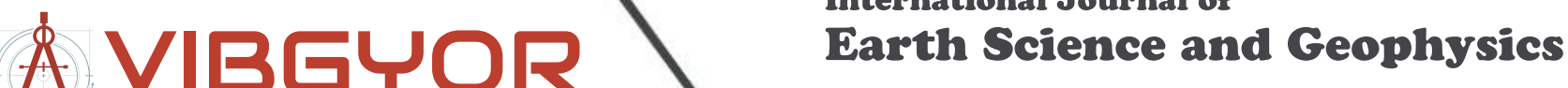

ISSN: 2631-5033

\section{Sentinel-1 SAR Data Revealing Fluvial Morphodynamics in Damghan (Iran): Amplitude and Coherence Change Detection}

\section{Tobias Ullmann ${ }^{1 *}$, Christian Büdel', Roland Baumhauer' and Majid Padashi',2}

${ }^{1}$ Physical Geography, Institute of Geography and Geology, University of Wuerzburg, Germany

${ }^{2}$ Geological Survey of Iran (GSI), Tehran, Iran

\begin{abstract}
The Sentinel-1 Satellite (S-1) of ESA's Copernicus Mission delivers freely available C-Band Synthetic Aperture Radar (SAR) data that are suited for interferometric applications (InSAR). The high geometric resolution of less than fifteen meter and the large coverage offered by the Interferometric Wide Swath mode (IW) point to new perspectives on the comprehension and understanding of surface changes, the quantification and monitoring of dynamic processes, especially in arid regions. The contribution shows the application of S-1 intensities and InSAR coherences in time series analysis for the delineation of changes related to fluvial morphodynamics in Damghan, Iran. The investigations were carried out for the period from April to October 2015 and exhibit the potential of the S-1 data for the identification of surface disturbances, mass movements and fluvial channel activity in the surroundings of the Damghan Playa. The Amplitude Change Detection highlighted extensive material movement and accumulation - up to sizes of more than $4,000 \mathrm{~m}$ in width - in the east of the Playa via changes in intensity. Further, the Coherence Change Detection technique was capable to indicate small-scale channel activity of the drainage system that was neither recognizable in the S-1 intensity nor the multispectral Landsat- 8 data. The run off caused a decorrelation of the SAR signals and a drop in coherence. Seen from a morphodynamic point of view, the results indicated a highly dynamic system and complex tempo-spatial patterns were observed that will be subject of future analysis. Additionally, the study revealed the necessity to collect independent reference data on fluvial activity in order to train and adjust the change detector.
\end{abstract}

Keywords

SAR, Radar, InSAR, Coherence, Iran, Geomorphology, Sentinel-1, Change detection

\section{Introduction and Objectives}

Fluvial activity is one of the most important actuating variables for the morphodynamics of arid regions. The fluvial events usually show a low frequency but a high magnitude and concentrate on strong single events in a particular time of the year. Consequently, these events can have major impact on the surface morphology and can modify the shape of the earth via erosion and accumulation processes. Seen from an anthropocentric point of view, such events can also imply negative consequences for land use activities, agricultural productivity or infrastructural services. It is of interest to quantify the temporal and spatial dimension of the events, the activity of channels and the material transportation and rearrangement: The scope is to further understand and comprehend the nature of arid fluvial systems.

Remote sensing techniques can partially provide information on these tasks: Several studies, like [1,2], have shown the effectiveness of spectral imaging by satellites for the characterization of surface characteristics and for change detection purposes. One further promising remote sensing tool to reveal fluvial morphodynamic in general and channel activity in particular is provided by imaging Synthetic Aperture Radar (SAR) Systems. Repeat SAR observations offer the application of interferometric analysis (via InSAR) and Coherence Change Detection (CCD). The CCD method quantifies the correlation between two SAR acquisitions during the InSAR processing. In opposite to the Amplitude Change Detection (ACD), the CCD is a coherent method and involves the entire information of the complex SAR signal - also the phase information [3-6]. CCD is known to be a very sensitive technique to reveal surface disturbances and changes on sub-pixel level and the technique has potential to indicate variations that are not detectable by multi-spectral, optical or SAR intensity data alone. Exemplarily [7] investigated this method for the arid region of the Salar de Uyuni Salt Lake, Bolivia, using ERS and Envisat ASAR data. Results pointed out that CCD is a promising way to contribute to the monitoring of fluvial activity. Further work that investigated the CCD for the detection of morphodynamic

*Corresponding author: Tobias Ullmann, Department Physical Geography, Institute of Geography and Geology, University of Wuerzburg, Am Hubland, D-97074 Wuerzburg, Germany, E-mail: tobias.ullmann@uni-wuerzburg.de

Received: January 29, 2016: Accepted: July 15, 2016: Published: July 18, 2016

Copyright: $\odot 2016$ Ullmann T, et al. This is an open-access article distributed under the terms of the Creative Commons Attribution License, which permits unrestricted use, distribution, and reproduction in any medium, provided the original author and source are credited. 
activity has, for example, been carried out by [8] who illustrated the potential of the CCD to detect movements of sand dunes in semi-arid zones of Niger with ERS SAR data. Using the X-Band instrument of the TerraSAR-X satellite [9] was able to map micro-morphological features in the arid environment of Palpa, Peru [5]. Investigated CCD and ACD with the TerraSAR-X satellite for the Bonneville Salt Flats, United States, and showed the applicability of the approach to map surface disturbance. Similar, [10] studied ERS data for repeat-pass InSAR and CCD of the Death Valley Salt Pan, United States. This type of SAR data was used as well by [11] for the detection of land surface changes in the Algerian desert. The authors reported the technique to be able to reveal the distribution of material transportation and erosion along river channels.

With the Sentinel-1 (S-1) satellite a SAR platform now operates that provides freely available C-Band SAR data suited for InSAR and CCD. The instrument offers a large coverage via the TOPSAR acquisition mode and is suited for analyzing extensive areas. Further, the S-1 Mission is planned to have a long operation time and the launch of additional S-1 twin-satellites makes a long consistency likely: the data will be suited to investigate processes and dynamics that take place on longer timescales, e.g., decades. These data point to new perspectives on the comprehension and understanding of surface changes and the monitoring of dynamic processes. The access is not restricted and not exclusive, but open for any user due to the free, full and open-data-policy. Studies providing explicit information about S-1 and the application of CCD were recently provided by $[12,13]$. The findings highlight the general applicability of the CCD approach and underline the advantage of the high temporal/spatial coverage that will be offered by the S-1 mission.

The objective of this research is to test the applicability of multitemporal S-1 IW SAR data in arid regions for the detection of surface changes induced by morphodynamic activity. It is of interest to examine if ACD is capable to reveal major events of surface alteration and to compare SAR and multispectral data. Further, it is an objective to test if CCD technique is sensitive to morphodynamic activity that is not visual in optical imagery or SAR intensity. The question is, if coherence can serve as an indicator for fluvial activity between two dates, e.g., to recognize if episodic or sporadic discharge events have happened. This contribution first highlights the environmental setting of the test site Damghan Playa, Iran. Afterwards the processing of the remote sensing data is shown and their properties are discussed. Section four presents the change detection techniques and discusses the argumentation to detect morphodynamic events with InSAR coherence. The results are shown and discussed for selected regions of the study area in the adjacent section. Finally, a brief summary is provided and conclusions are drawn.

\section{Test Site - Damghan Playa}

The test site is located in northeastern Iran in the Semnan province, close to the settlements Damghan and Shahrud. The region is surrounded by the mountains and foothills of the North Alborz Mountains that belong to the Alp-Himalaya orogenic belt. The elevation of the site can climb up to more than 2,000 meter and the basin of the central Damghan Playa lies above 1,000 meter altitude. The climate of the region is characterized as cold dessert climate $(\mathrm{BWk})$ with an average yearly temperature of about $15^{\circ} \mathrm{C}$ and a precipitation of $200 \mathrm{~mm}$ [14]. The climate of the site shows distinct summer and winter situations. The hottest and driest conditions are met between May and August. The period between September and April is characterized by cooler and wetter conditions. The region is not covered by natural vegetation, but bare soils, salt deposits and hard rock are exposed. The small agricultural land use activities concentrate on the surroundings of the settlements Damghan and Shahrud. The active morphodynamics are mainly driven by aeolian and fluvial activities; the one named last concentrate on the winter half year. The fluvial activities are prominent as slop deposits, alluvial domains and mega fans.

Damghan Playa, which is named by locals Kavir-e-Haj Aligholi, is one of the biggest playas in southern flank of the Alborz Mountains, composed of alluvial fan deposits, saline lake and aeolian deposits [15]. The lake is covered vastly by salt polygons in different size and thickness and there is a bright gray cover in the central part of lake that may have been resulted by the aeolian activities. Few months every year the water table may rise above the ground and the playa is flooded- a result of precipitation events. The alluvial deposits in the northern rim are cut by young faults and caused a step of approximately three meters. This slope exposes the bright, sandy and silty sediments of the lake. Figure 1 indicates the location of the site and further shows the elevation (Figure 1a), the average S-1 intensity (Figure 1b) and a spectral true-color RGB composite (Figure 1c). The sedimentary history and the geomorphological setting of the site were subject of field work in the years 2013 and 2014, conducted by the Department of Physical Geography of the University of Wuerzburg, Germany, and the Geological Survey of Iran.

\section{Database and Pre-Processing}

Two datasets were used in this study to examine the potential to detect short term morphodynamic processes in the surroundings of Damghan Playa using remote sensing imagery. On the one hand multispectral data of the Landsat-8 (L-8) satellite operated by NASA were obtained. On the other hand Sentinel-1 SAR radar data were provided by ESA. Both satellites provide regularly observations on the earth's surface and are freely available via the Earth Explorer [16] and the Sentinels Scientific Data Hub [17]. Table 1 provides an overview on the remote sensing data used for this study and lists the key parameters of each dataset. These data were interpreted in context of the weather history and data on the temperature, the precipitation and weather events were obtained from a nearby climate station. Further, the digital elevation model of the SRTM Mission was used to characterize the topographic and hydrographic setting of the site.

\section{Sentinel-1 SAR data}

The first Sentinel-1 satellite (S-1a) was launched on 30.04.2014, is part of ESA's Copernicus Mission and operated by the European Space Agency (ESA). The SAR instrument used is a C-Band radar system at $5.405 \mathrm{GHz}$ that delivers single- or dual-polarized data. The operational modes offer wide spectra concerning the coverage and resolution of a single acquisition, e.g., the interferometric wideswath TOPSAR mode (IW) offers a swath of $250 \mathrm{~km}$, with a ground resolution of less than 20 meters. The revisit time of the $S-1$ is 12 days, but will be 6 days once a two-satellite constellation is operational. S-1a is planned to run at least seven years; the launch of the twin satellite S-1b is scheduled for 2016 [18,19]. In December 2015 contracts were signed to continue with S-1c and S-1d in near future. Figure $1 \mathrm{~b}$ shows the temporally averaged $S-1$ intensity of the test site.

For this study S-1 SAR data were downloaded from ESA'S Sentinels Scientific Data Hub (ESA, 2016) as Ground Range Detected (GRD) and Single Look Complex (SSC) IW products. Only data with 24 or 48 days time-lag were available, in opposite to the nominal revisit time of 12 days. The GRD data display the focused, calibrated, geocoded and multi-looked Level-1 intensities without phase information. No further processing was applied to the GRD products. The comparability between the dataset is given since all data were recorded with the same acquisition geometry (orbit and incidence angle) and underwent the same processing/calibration at the operator's SAR processor. The interferometric processing of the SSC data was done using ESA's Sentinel Application Platform (SNAP) and the steps of the workflow are illustrated in figure 2 (left). The SRTM DEM data were used for the back coding. The Flat-EarthPhase was removed using the standard settings of the polynomial 

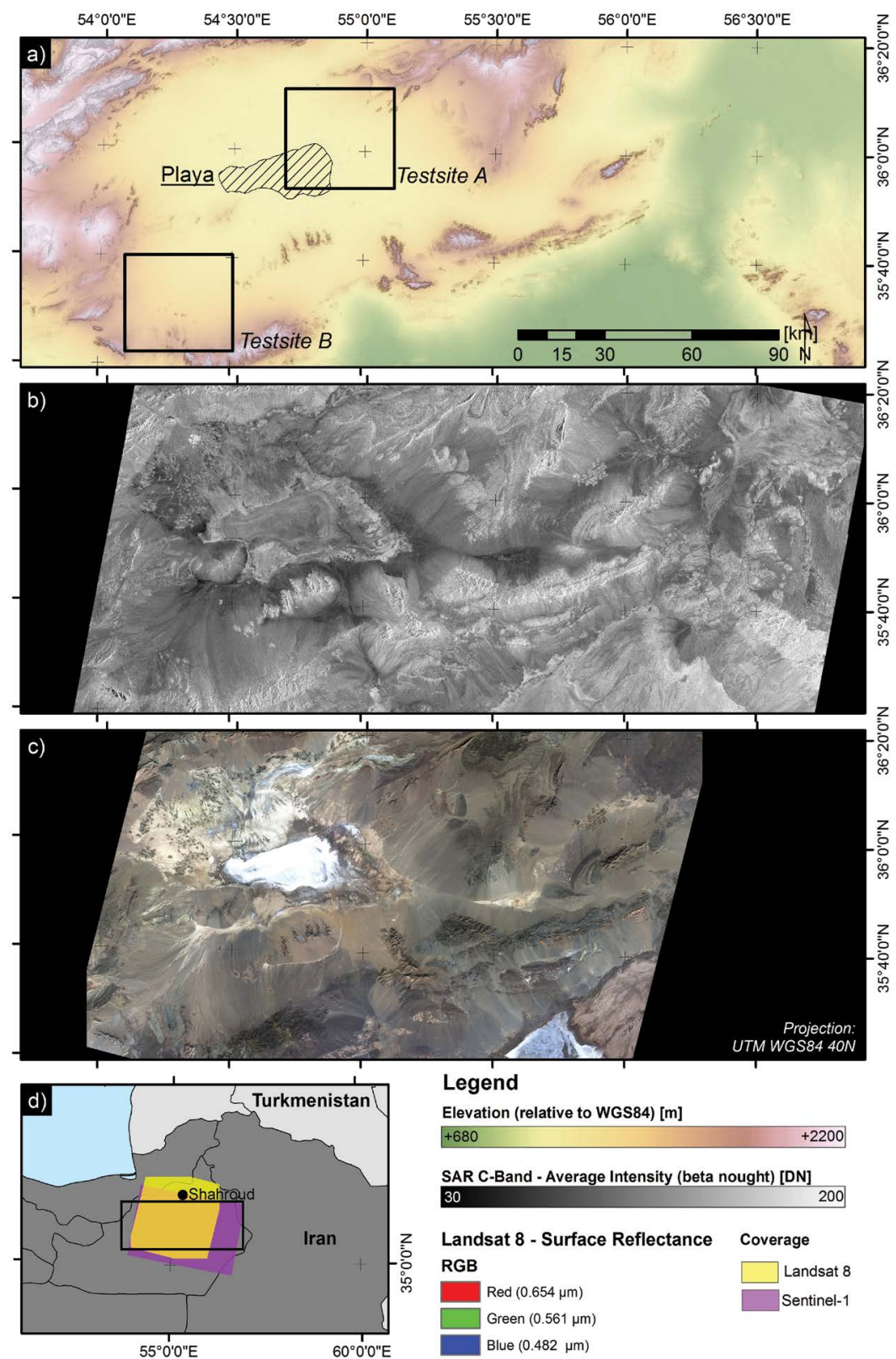

Legend

Elevation (relative to WGS84) [m]

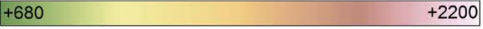

SAR C-Band - Average Intensity (beta nought) [DN]

Landsat 8 - Surface Reflectance Coverage

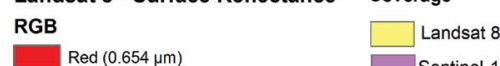

Red $(0.654 \mu \mathrm{m})$
Green $(0.561 \mu \mathrm{m})$
Blue $(0.482 \mu \mathrm{m})$

Figure 1: Location of the site damghan. a) SRTM DEM elevation and locations of test sites A and B; b) average Sentinel-1 intensity; c) true color RGB composite of Landsat-8 acquired on 02. May 2015; and d) over view map and coverage of the remote sensing data.

Table 1: Database and key acquisition parameters of Sentinel-1(S-1) and Landsat-8 (L-8).

\begin{tabular}{|c|c|c|c|c|c|c|c|}
\hline Sensor & Date & Path/Orbit & Resolution & Bands & Scene Center Incidence Angle & Sun Elevation & Sun Azimuth \\
\hline S-1 & 02.05 .2015 & 64 & $15 \mathrm{~m}$ & VV & $33.907^{\circ}$ & $n / a$ & $\mathrm{n} / \mathrm{a}$ \\
\hline S-1 & 26.05.2015 & 64 & $15 \mathrm{~m}$ & VV & $33.906^{\circ}$ & $\mathrm{n} / \mathrm{a}$ & $n / a$ \\
\hline S-1 & 19.06.2015 & 64 & $15 \mathrm{~m}$ & VV & $33.908^{\circ}$ & $\mathrm{n} / \mathrm{a}$ & $\mathrm{n} / \mathrm{a}$ \\
\hline S-1 & 30.08 .2015 & 64 & $15 \mathrm{~m}$ & VV & $33.912^{\circ}$ & $\mathrm{n} / \mathrm{a}$ & $\mathrm{n} / \mathrm{a}$ \\
\hline S-1 & 17.10 .2015 & 64 & $15 \mathrm{~m}$ & VV & $33.920^{\circ}$ & $\mathrm{n} / \mathrm{a}$ & $\mathrm{n} / \mathrm{a}$ \\
\hline L-8 & 19.05.2015 & P162/R35 & $30 \mathrm{~m}$ & $0.44-2.20 \mu \mathrm{m}$ & $\mathrm{n} / \mathrm{a}$ & $65.85^{\circ}$ & $127.14^{\circ}$ \\
\hline L-8 & 07.08 .2015 & P162/R35 & $30 \mathrm{~m}$ & $0.44-2.20 \mu \mathrm{m}$ & $\mathrm{n} / \mathrm{a}$ & $62.08^{\circ}$ & $128.59^{\circ}$ \\
\hline L-8 & 08.09 .2015 & P162/R35 & $30 \mathrm{~m}$ & $0.44-2.20 \mu \mathrm{m}$ & $\mathrm{n} / \mathrm{a}$ & $54.42^{\circ}$ & $143.43^{\circ}$ \\
\hline L-8 & 26.10 .2015 & P162/R35 & $30 \mathrm{~m}$ & $0.44-2.20 \mu \mathrm{m}$ & $\mathrm{n} / \mathrm{a}$ & $39.04^{\circ}$ & $158.56^{\circ}$ \\
\hline
\end{tabular}

estimation function. After interferogram generation, the coherence of the coregistered data was calculated using a moving window with a size of nine by nine pixels. After the deburst and the merge of the three sub-swaths, the coherences were resampled to a resolution of 14 meter (ground range) using cubic interpolation. Finally, the SRTM
DEM data were used for the Range-Doppler Terrain Correction. The coherences were geocoded to the UTMWGS84 Zone 40 North coordinate system.

After the processing the data were visually inspected but did not show noticeable anomalies in along- or across-track direction, e.g., 

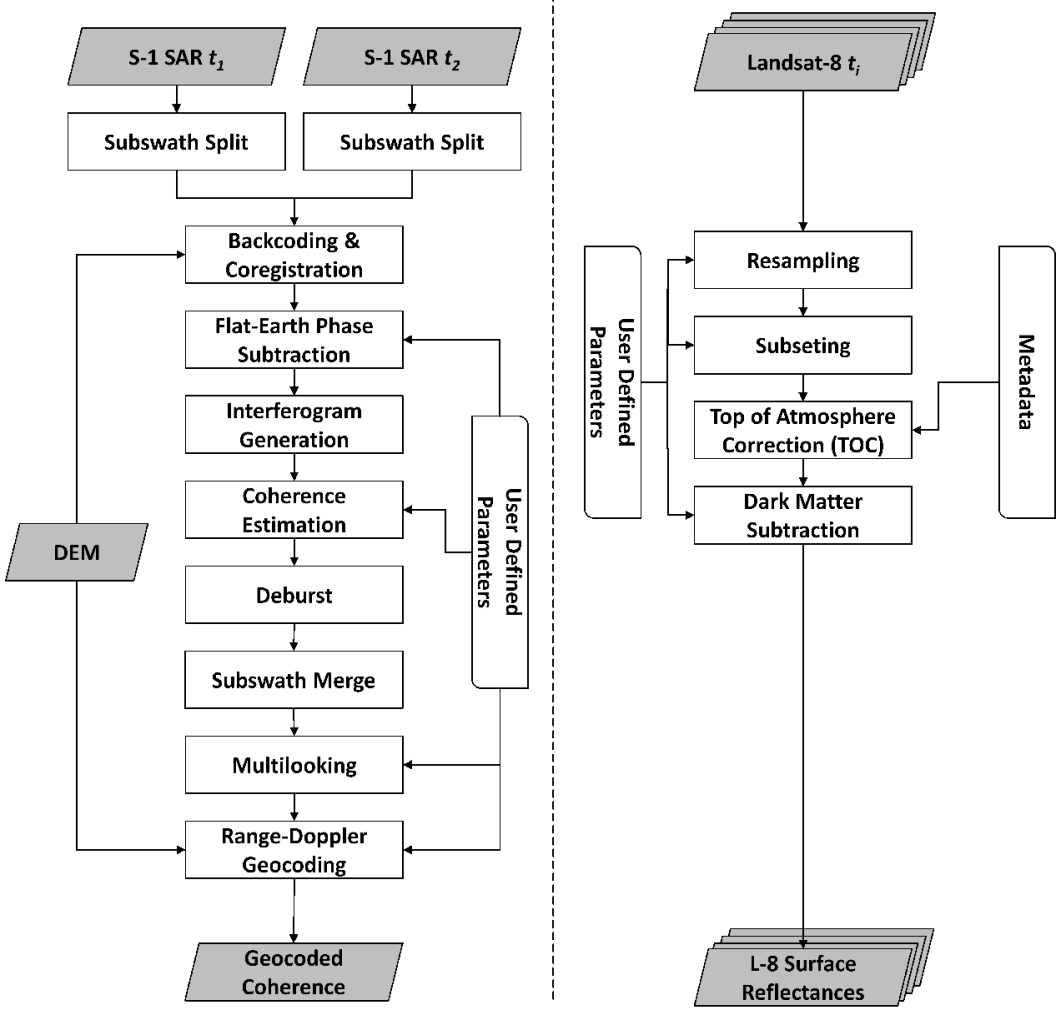

Figure 2: General processing of Sentinel-1 SAR data (S-1) (left) and Landsat-8 multispectral data (right).

Table 2: Statistical parameters of Sentinel-1 coherences between 02 May 2015 and 17 October 2015.

\begin{tabular}{|c|c|c|c|c|c|c|c|c|}
\hline ID Coherence & Master & Slave & Time Lag [d] & Median & Mean & Standard Deviation & Percentile 1\% & Percentile 99\% \\
\hline \multicolumn{9}{|l|}{ a) Entire Site } \\
\hline IDC -1 & 02.05 .2015 & 26.05 .2015 & 24 & 0.64 & 0.609 & 0.156 & 0.174 & 0.848 \\
\hline IDC -2 & 26.05 .2015 & 19.06 .2015 & 24 & 0.768 & 0.727 & 0.167 & 0.167 & 0.932 \\
\hline IDC -3 & 19.06.2015 & 06.08 .2015 & 48 & 0.746 & 0.69 & 0.192 & 0.128 & 0.921 \\
\hline IDC -4 & 06.08.2015 & 30.08 .2015 & 24 & 0.773 & 0.72 & 0.166 & 0.171 & 0.914 \\
\hline IDC -5 & 30.08 .2015 & 17.10 .2015 & 48 & 0.74 & 0.695 & 0.191 & 0.132 & 0.933 \\
\hline \multicolumn{9}{|l|}{ b) Testsite A } \\
\hline IDC -1 & 02.05 .2015 & 26.05 .2015 & 24 & 0.627 & 0.6 & 0.157 & 0.172 & 0.848 \\
\hline IDC -2 & 26.05 .2015 & 19.06 .2015 & 24 & 0.728 & 0.691 & 0.159 & 0.219 & 0.913 \\
\hline IDC -3 & 19.06.2015 & 06.08 .2015 & 48 & 0.646 & 0.615 & 0.202 & 0.12 & 0.903 \\
\hline IDC -4 & 06.08.2015 & 30.08 .2015 & 24 & 0.7 & 0.637 & 0.207 & 0.099 & 0.895 \\
\hline IDC -5 & 30.08 .2015 & 17.10 .2015 & 48 & 0.676 & 0.625 & 0.201 & 0.102 & 0.904 \\
\hline \multicolumn{9}{|l|}{ c) Testsite B } \\
\hline IDC -1 & 02.05 .2015 & 26.05 .2015 & 24 & 0.654 & 0.637 & 0.122 & 0.276 & 0.84 \\
\hline IDC -2 & 26.05.2015 & 19.06.2015 & 24 & 0.731 & 0.707 & 0.118 & 0.294 & 0.884 \\
\hline IDC -4 & 06.08 .2015 & 30.08 .2015 & 24 & 0.664 & 0.644 & 0.165 & 0.227 & 0.896 \\
\hline IDC -5 & 30.08 .2015 & 17.10 .2015 & 48 & 0.724 & 0.701 & 0.152 & 0.254 & 0.913 \\
\hline
\end{tabular}

no systematic decreases towards the far- or near-ranges of the subswaths. Further, no atmospheric interferences or artifacts were visual in the interferograms. However, for all of the final coherences and interferograms we noticed a gap of several pixels between some parts of the sub-swaths; none of these gaps was crossing the areas of interest. However, additionally, some regularly spaced strips were visual close to the settlement of Shahrud. The direction of these artifacts is random and may presumptively be a result of the local ground radar system of the neighboring airport (Shahrud Airport RUD). The appearance of this anomaly was restricted and did only partially affect the area of interest. In total five coherences were calculated using the available six S-1 datasets. Table 2 lists the data used for the derivation of the coherences, the master and slave configuration, the time lags between the acquisitions and the general statistical parameters on the quality of the coherence. For all of the data it can be noted that the mean coherence was high and around 0.7 with an average standard deviation of 0.165 , no matter if the time lag was 24 or 48 days. Thus, the data showed satisfying quality and the assessment indicated general applicability of coherence analyses.

\section{Landsat-8 multispectral data}

The Landsat mission is operated since 1972 and is continued our days using the eighth Landsat satellite. L-8 carries two instruments which are the Operational Land Imager (OLI) and the Thermal Infrared Sensor (TIRS). The OLI is a multispectral system that is able to acquire ninespectral bands in the range from $0.44 \mu \mathrm{m}$ to $2.20 \mu \mathrm{m}$. The revisit time is 16 days and $\mathrm{L}-8$ provides coverage of approximately $170 \mathrm{~km}$ by $180 \mathrm{~km}$ per scene and with $30 \mathrm{~m}$ spatial resolution (OLI) [20]. Figure 1c exemplarily shows an L-8 true color RGB composite of the test site. 
The L-8 data were processed using Exelis' ENVI5.3 and IDL 8.4. The general processing workflow is illustrated in figure 2 (right). As a first step the data were cropped and resampled to a common grid with subpixel accuracy using bilinear interpolation. The target coordinate system was UTMWGS84 Zone $40 \mathrm{~N}$ and thus matches the S-1 data. In the following the data were corrected to top of atmosphere reflectance using the provided metadata. Finally, the dark subtraction [21] was applied in order to remove haze and the blue shift. The data sets were now expressed as surface reflectances in the range from zero to one. All L-8 datasets were visually inspected afterwards but did not show any noticeable anomaly, e.g., induced by atmospheric effects ortho erroneous processing. Further, the selected data were cloud and snow free.

\section{Climate data}

Data on the weather history are of high importance, since SAR imagery and InSAR coherences are highly affected by changing ground moisture conditions. The Shahrud Airport lies very close to the test sites and publishes the Meteorological Aviation Routine Weather Report (called METAR) on daily bases [22]. The location of Shahrud is marked in figure $1 \mathrm{~d}$ and the distance to test site $\mathrm{A}$ is approximately $40 \mathrm{~km}$. Exemplarily, the METAR report includes information on the mean, minimum and maximum daily air temperature, the daily accumulated amount of precipitation and further provides nominal information on weather events, e.g., rainfall, or storms. Figure 3 shows the climate data of the station between April and October 2015 and the acquisition times of L- 8 and S-1. The temperature data showed a positive trend from April to July and a negative trend from August to October 2015. In general, rainfall and storm events were more frequent in spring and autumn; however, some strong events happened during the summer months. A strong rainfall event with thunderstorms and strong cooling happened on 20.07.2015, between the S-1 acquisitions taken on 19.06 and 06.08.2015. The corresponding coherence of these time span is IDC-3. Further relevant rainfall events took place on 22.08, 01.09, 17.09, 09.10 and
17.10.2015. The corresponding coherences of these dates are IDC-4 (06.08 - 30.08.2015) and IDC-5 (30.08 - 17.10.2015).

\section{Methodology}

The imaging with SAR Systems is based on the active sending and receiving of electromagnetic pulses in the microwave spectrum (approximately in the range from 1 to $20 \mathrm{GHz}$ ). The electromagnetic waves are defined by the power (intensity/amplitude), the phase and the direction of propagation, the polarization and the velocity. Generally, the received and send signal show differences: these are predominantly caused by the interaction of the wave with the object of study (target), respectively the earth's surface. The microwave radiation can transit objects depending on the properties of the wave and of the target. Most important factors that alter the signal are the dielectric and geometric properties of the target [23]. The generation and interpretation of SAR imagery is therefore immanent different from spectral/optical imagery and it is less intuitive. However, an advantage compared to optical imagery is that SAR system are nearly unaffected by cloud cover and that they do not depend on external illumination (sun light) [23]. Additionally, such active systems provide a high consistency of the signals: Coherent SAR affords a reliable source of information for monitoring purposes and has shown to be of value for change detection and time series analysis $[4,5]$.

Two types of change detection techniques can be distinguished, subject to the condition that pre- and post-event SAR data were acquired with the same geometry and system configuration: the (incoherent) Amplitude Change Detection (ACD) and the Coherent Change Detection (CCD) [6]. Both approaches were investigated in order to estimate the potential of S-1 to identify of surface disturbances, mass movements and fluvial activity.

\section{SAR amplitude change detection}

The most native way to detect surface changes between two dates/ acquisitions is the comparison of the SAR intensities, respectively

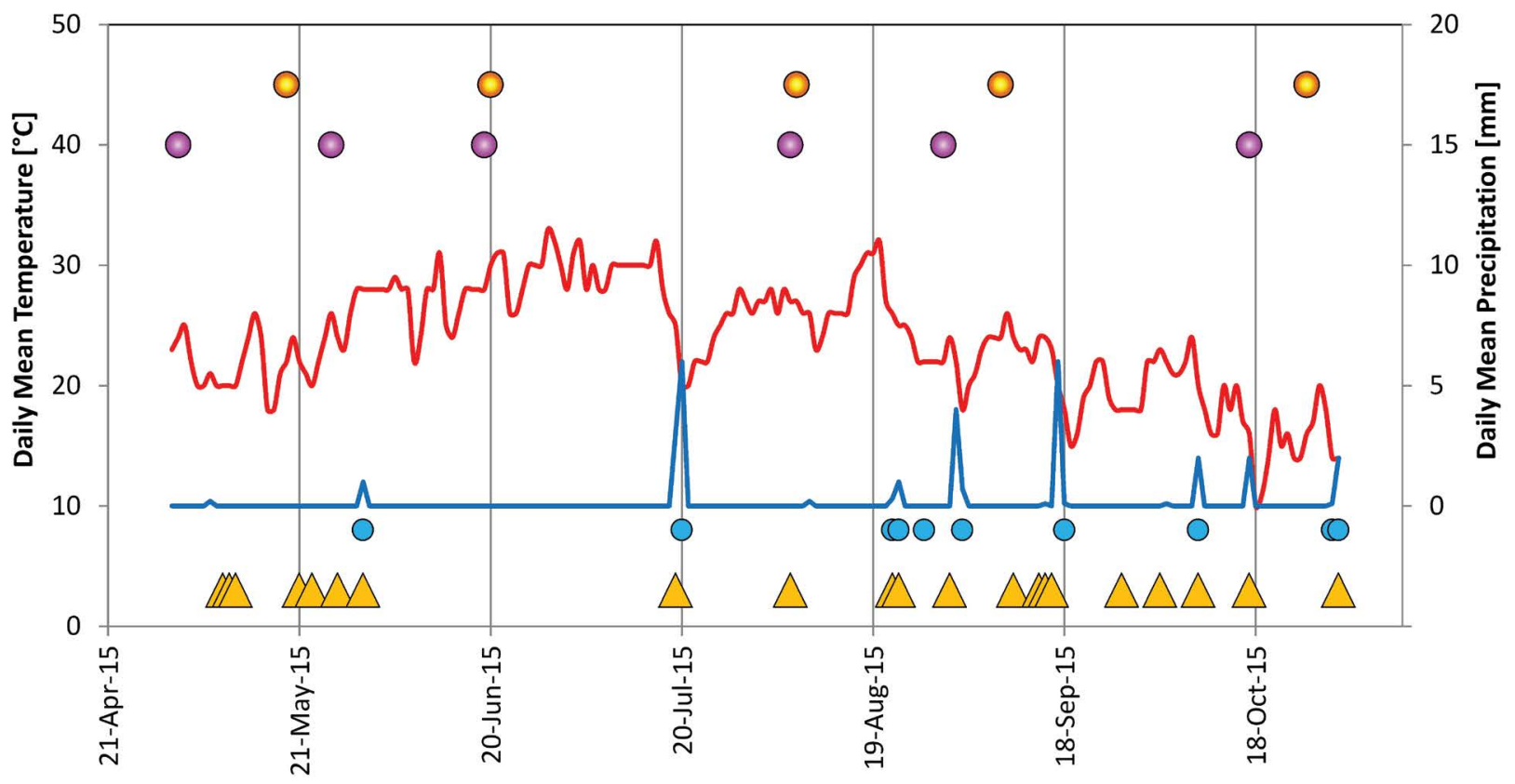

—Daily Mean Temperature $\left[{ }^{\circ} \mathrm{C}\right]$ O S-1 Acquisition

L-8 Acquisition

—Precipitation $[\mathrm{mm}] \triangle$ Storms $\bigcirc$ Rainfall
Station Coordinates: $36^{\circ} 25^{\prime} \mathrm{N} 55^{\circ} 01^{\prime} \mathrm{E}$-- Station Altitude: $1345 \mathrm{~m}$

Figure 3: Climate data of the station Shahrud between April and October 2015, storm and rainfall events and acquisition dates of Sentinel-1 (S-1) and Landsat-8 (L-8). Source: METAR weather report Shahrud Airport (RUD). 
to compare the intensities of the reference and the test image. This technique is only sensitive to changes that affect the back scatter and does not take the entire complex SAR information (intensity and phase) into account [24]. Events that may be detected with ACD are for example extensive floods, or mass movements. However, such events must have a sufficient large size to be recognizable in the SAR imagery and the changes in intensity must be higher than the noise or speckle effect.

\section{SAR coherence change detection}

A more sensitive technique to detect changes between two SAR acquisitions is the CCD. This method involves also the phase information and is therefore capable to reveal changes that are not visual in the intensities/amplitudes. Two SAR acquisitions might indicate the same intensity, however, changing surface conditions may have led to phase differences and therefore to a decorrelation of the complex signals. Further, CCD is sensitive to changes on subpixel scale, e.g., changes smaller than one resolution cell. Coherent methods can be applied for the change detection, since interferometric analysis implicates the formation of the complex interferogram. The phase of the interferogram is sensitive to terrain elevation and ground motion, e.g., to heave or subsidence. Beside this the interferogram contains information about the correlation of the two SAR scenes. The correlation coefficient $\gamma$ for two (zero-mean) complex signals $-s_{1}, s_{2}$ - is defined as follows according to [25] (1):

$$
\gamma=\frac{E\left\{s_{1}^{*} \cdot s_{2}\right\}}{\sqrt{E\left\{\left|s_{1}\right|^{2}\right\} \cdot E\left\{\left|s_{s}\right|^{2}\right\}}}
$$

Where $E\left\{s_{1}^{*} \cdot s_{2}\right\}$ is the expectation value; $s_{1^{*}}, s_{2}$ are the backscattered signals and $s_{1}^{*}$ describes the complex conjugate of $s$. The dimensionless correlation coefficient $\gamma$ describes the quality of the interferometric phase. Its values range from zero, which means no correlation, to one, which indicates full correlation. The correlation coefficient $\gamma$ is affected by several factors as shown in the following (2) and as described by [3]

$$
\gamma=\gamma_{\text {temp }} \cdot \gamma_{S N R} \cdot \gamma_{\text {geo }} \cdot \gamma_{\text {vol }}
$$

$\gamma_{\text {temp }}$ denotes temporal correlation, as well as any changes of the surface conditions between the two data acquisitions. $\gamma_{S N R}$ expresses the correlation that is caused due to thermal, instrument and processor noise. $\gamma_{\text {geo }}$ quantifies the influences on $\gamma$ caused by the geometrical/ spectral correlation. Finally, $\gamma_{v o l}$ involves the effect of volume backscattering that cause a decorrelation of the signals [3]. Main reasons for the decorrelation via $\gamma_{\text {temp }}$ are for example: anthropogenic changes, land use activities, changing plant physiology, changes in the ground moisture regime, or displacement [6]. Any change of the surface or morphology can therefore be addressed to $\gamma_{\text {temp }}$. The variable is as well the dominant factor altering the coherence in repeat pass CCD [6].

In the InSAR processing the correlation coefficient $\gamma$ can be estimated directly from the data. The sample coherence $\hat{\gamma}$, shown in (3), can be used for the estimation. The derivation follows the assumption that the processes involved are in mean ergodic; therefore spatial averaging is necessary and $N$ represents the size of the (spatial) estimation window. The topographic phase $\phi_{T}$ is compensated which is of importance in mountainous and rough terrain [6].
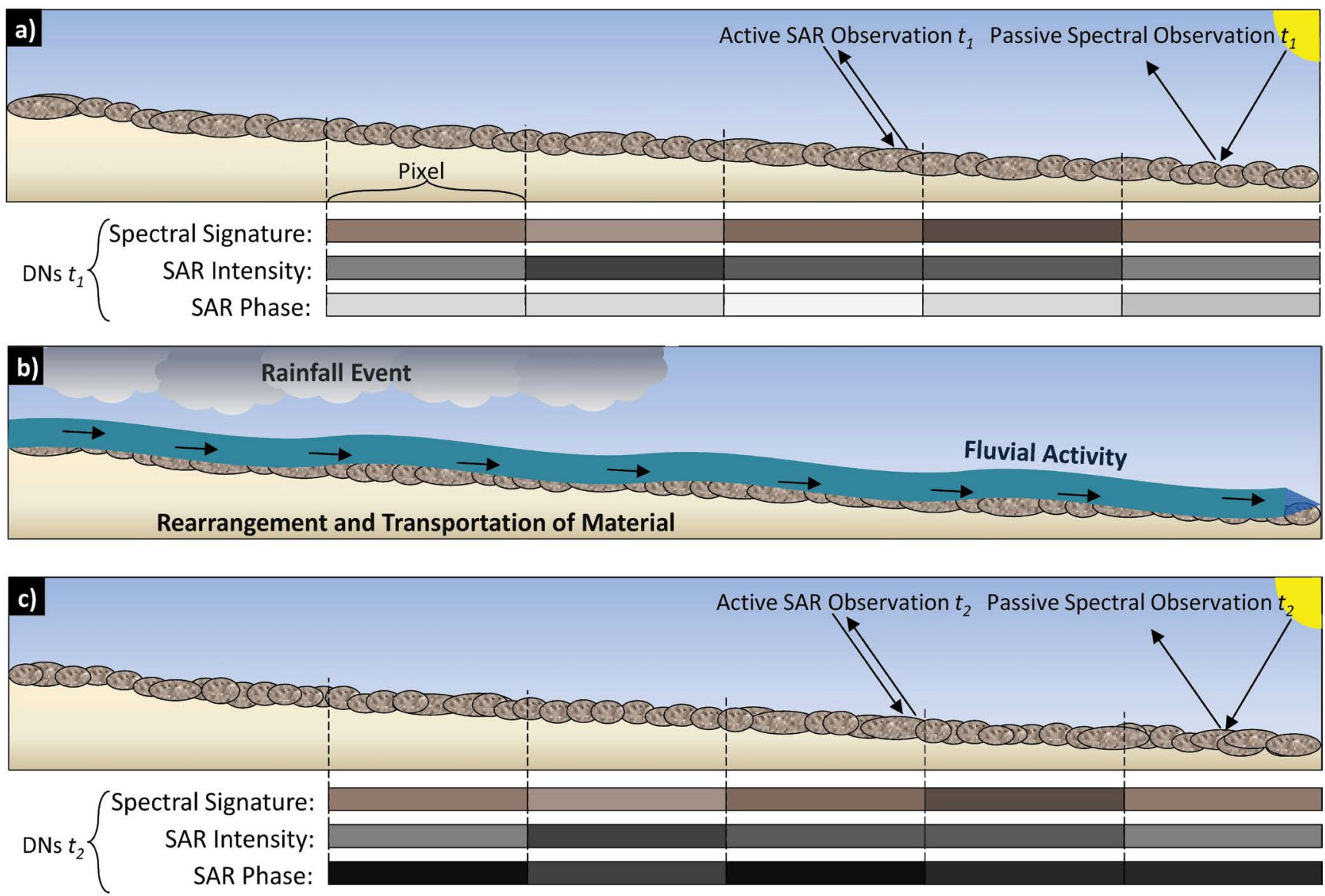

Figure 4: General schema on the change detection. a) pre-event situation; b) rainfall event, fluvial activity and material transportation; c) post-event situation with rearranged material. The Digital Numbers (DNs) of the spectral signatures and SAR intensities remained unchanged between $t_{1}$ and $t_{2}$. contrary, the SAR phases had changed due to the rearrangement of material. 


$$
\hat{\gamma}=\frac{\sum_{n=1}^{N} s_{1}^{*(n)} \cdot s_{2}^{(n)} \cdot e^{-j \phi_{T}}}{\sqrt{\sum_{n=1}^{N}\left|s_{1}^{(n)}\right|^{2} \cdot \sum_{n=1}^{N}\left|S_{2}^{(n)}\right|^{2}}}
$$

The sample coherence $\hat{\gamma}$ is a sensitive quantity if the other factors controlling the correlation coefficient $-\gamma_{S N R} \cdot \gamma_{g e o} \cdot \gamma_{v o l}$ - are constant, respectively negligible. For arid natural non-vegetated regions one can expect $\gamma_{v o l}$ to be of minor relevance, since vegetation serves as the most important source for natural volume scattering and its absence favors high coherence. Further, influences of changing ground moisture will only be present after rainfall events due to the arid nature of the site. The assessment provided in table 2 indicates the high coherence values of all test sites and therefore the general feasibility of the CCD. It is assumed that $\gamma_{\text {temp }}$ is the dominant factor controlling the sample coherence $\hat{\gamma}$.

The approach to be tested here is if the analysis of repeat pass CCD is capable to reveal changes on the activity of fluvial systems of arid regions. Thus, it is of interest if the coherence can serve as an indicator of channel activity between two dates, e.g., when short term episodic or sporadic discharge events happened. Such activity will not necessarily be noticeable in optical, multispectral or SAR intensity imagery. However, most likely the transportation and rearrangement of material will cause changes in the phase and therefore will also cause a decorrelation between the reference and the test image, which is schematically illustrated in figure 4 . This means that active channels and drainage systems will be highlighted by lower coherence $\hat{\gamma}$ compared to the inactive parts of the drainage systems and the surroundings uplands.

\section{Results}

This approach was investigated for the two test sites A and B (Figure 1). The time series data of S-1 (intensity and coherence) and of L-8 (OLI) were visually analyzed and changes of the features were qualitatively interpreted in context of the weather data and the topographic setting. Additionally, a quantitative assessment was conducted. The characteristics of L-8 data, the intensities (ACD) and the SAR coherences (CCD) were analyzed over time for the active parts of the drainage system in test site $B$.

\section{Visual analysis}

Figure 5 shows the situation of the eastern side of Damghan Playa between the 19.06 and the 17.10.2015. Between the first two dates no major surface changes were recognizable - neither in the L-8 data, nor in the SAR intensity. The coherences (IDC-2 \& IDC-3) indicated some changes of the surface properties which were mainly restricted to the foot slope of the flank and the eastern rim of the playa. The coherence values were high and around 0.6 to 0.9 for most parts of the subset. Taking the weather data into account (compare Figure 3), it seemed that the rainfall event of the 20.07.2015 did not cause recognizable activity in this area, at least none that was visual in the data sets. Contrary, major flow activity was visible in all of the data sets for the time span between the 06.08 and the 30.08 .2015 and this activity could be associated with the rainfall and storm events that happened during this period. The L- 8 data sets indicated that material was transported on the playa that was much darker than the salty bright deposits. The same changes were visible via lower intensity values in the SAR data. The coherence IDC- 4 highlighted the flow activity and the major flow paths decorrelated between the acquisitions (marked with the black arrows) while the surrounding remained coherent. The activity intensified between the 30.08 and the 17.10.2015 and L-8 and S-1 showed changes in the spectral characteristics and the intensity. The flow widened and additional surface changes were recognizable in the southeastern, eastern and central part of the subset (black arrow). These were well expressed in the coherence (IDC-5) and the flow lines showed low coherence values. The observed surface changes were associated with the rainfall and storm events that happened during this period, however, information on the spatial extent of the events were not available.

The data sets were investigated for test site B and for the same period (Figure 6). The time series of the L-8 data sets showed only some changes that were visually recognizable. Most noticeable was the higher surface reflectance between the 19.06 and the 06.08.2015 of the drainage channel that crossed the area from southwest to northeast. The SAR intensities showed more variations of the brightness values over time, but the visual recognition was difficult and less clear compared to the situation in test site A. Most noticeable was the change of the SAR intensity of the north-central part of the subset where the streamlines converge at the local foot slope. The analysis of the coherences provided a more detailed picture and IDC-2 (first coherence shown in the Figure 6) indicated a drop of the coherence inside the active part of the drainage system (black arrow). The values were low and about 0.2 , compared to high coherence values of around 0.7 to 0.9 of the inactive parts and the surrounding uplands. IDC-3 showed a different situation and this channel was most likely not active between the 19.06 and the 06.08.2015, but the drainage systems in the central eastern part of the subset (black arrow). An extensive loss of the coherence between the SAR signals was then observed for IDC-4 between the 06.08 and the 30.08.2015. The identification of active channels was less clear due to the general low coherent environment of northern part of the area. Remarkable was a sharp line between high coherence in the southern part and the low coherence in the northern part. This separation was as well visible in IDC-5 and it ran along with the contour lines. Further, IDC5 indicated major activities in the lower part of the area close to the foot slope (black arrow).

Figure 7 provides a summary on the surface changes that were visually detected between the 19.06 and the 17.10.2015. The changes recognized in both test sites were manually digitized between the shown boundaries (dashed black lines). The S-1 coherences and intensities served as basis for the digitalization and elevation data of the SRTM DEM served as reference for the interpretation of flow activity. For the test site A it was clear that the downstream runoffs were of high magnitude and that the streams widened once they reached the low-lying flat basin (Figure $7 \mathrm{a}$ ). The two central flows recognized in IDC- 4 and IDC- 5 reached the surface of the playa and showed lobes of more than $4,000 \mathrm{~m}$ in width. The high transportation capacity was a result of the topographic situation. The flows had large catchment areas due to their localization at the end of the valley. The figure highlights as well the different spatial patterns of activity. Between the 06.08 and the 30.08.2015 (IDC-4) the northern part of the area was more active, while between the 30.08 and the 17.10.2015 (IDC-5) the southern part showed higher activity. Figure $7 \mathrm{~b}$ provides the digitized activity based on IDC-2 to IDC-5for test site B. The varying activity of channels highlighted the high dynamic of the drainage system. Some channels were used more frequent than others during the observation period.

Additionally, the identified surface changes were compared to the potential flow accumulation and the channel network. This information was derived via hydrographic modeling from the SRTM DEM data [26]. Figure 7c shows the flow accumulation of test site A calculated with the multi-flow-direction approach (MDF) [27]. The MFD approach allows flows to converge and diverge. The more flows converge the higher is the accumulation and the value of the feature. The comparison to the digitized surface changes showed that the runoffs detected in IDC- 4 and IDC- 5 were partially congruent with the modelled flows. However, major dissimilarities between the modelled and observed fluvial activity were observed for the lowlying flats and the playa. Figure $7 \mathrm{~d}$ shows the flow accumulation of test sites B. The drainage network was characterized by various converging and diverging channels that finally meet at the foot slope close to the northern digitalization boundary. The general shape of this drainage network was observed in the coherences and the active 

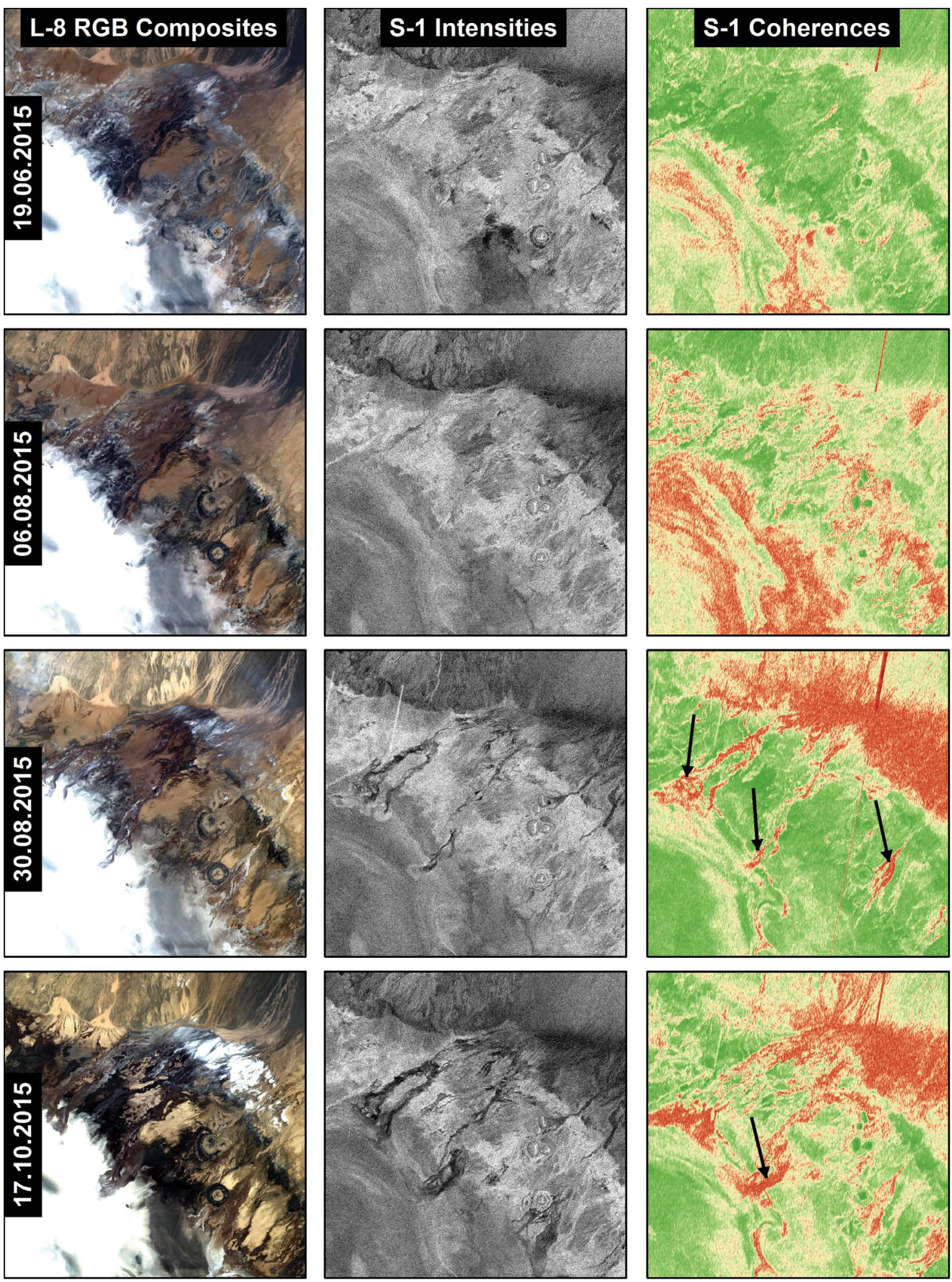

\section{L-8 Surface Refelctances}

Truecolor Composite

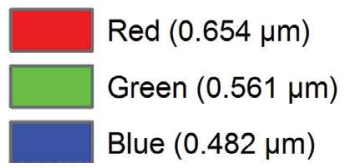

\section{S-1 Intensity [DN]}

30

\section{S-1 Coherence}

\section{0}

200

Projection: UTM WGS84 40N A

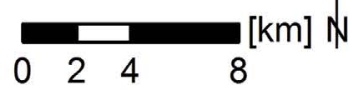

Figure 5: Landsat-8 (L-8) true color spectral reflectances, Sentinel-1 (S-1) SAR intensities and coherences for the period from June to October 2015 for test site A. 

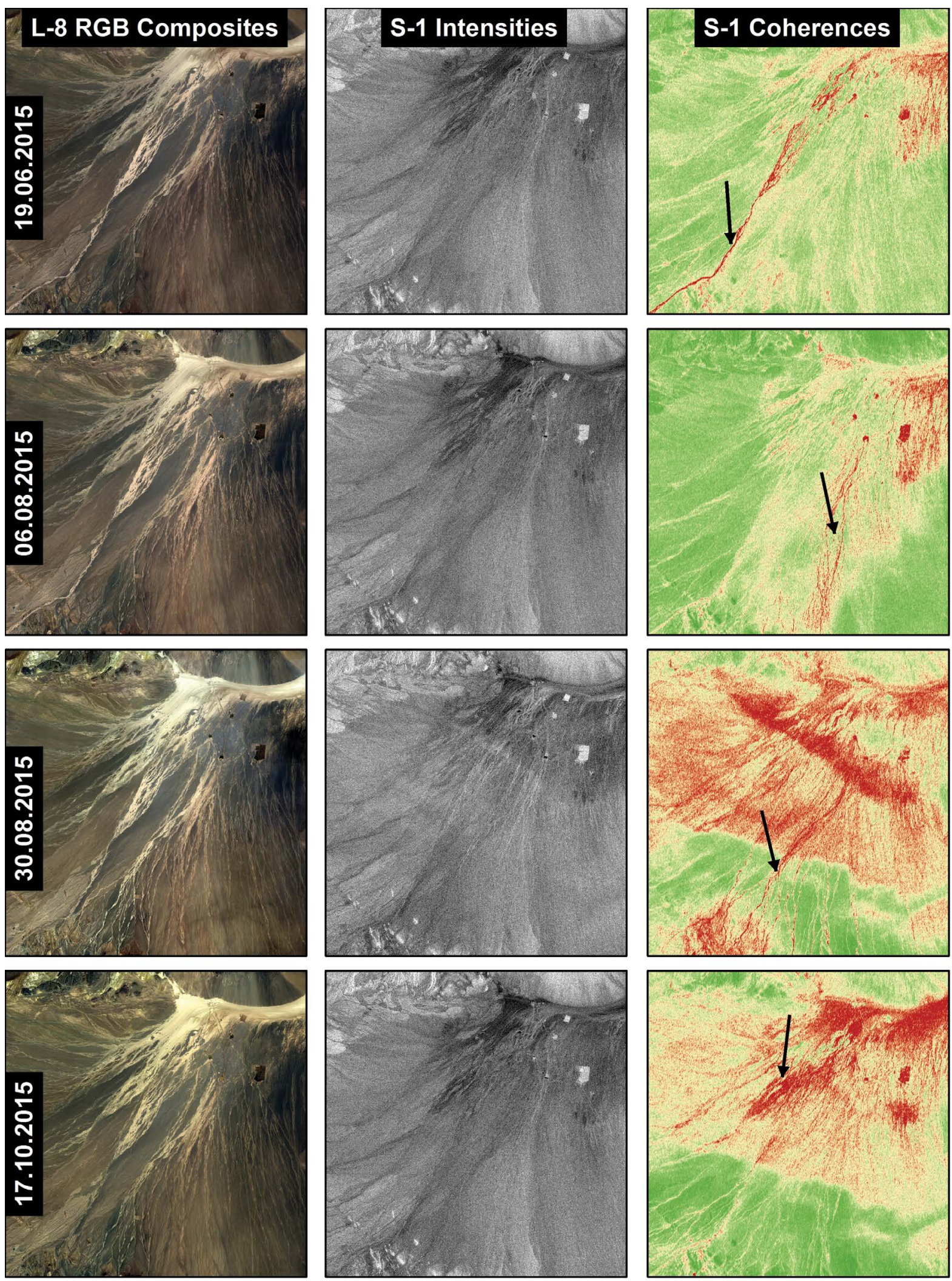

L-8 Surface Refelctances

Truecolor Composite

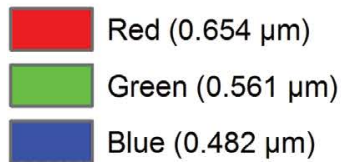

\section{S-1 Intensity [DN]}

30

\section{S-1 Coherence}

\subsection{5}

200

Projection: UTM WGS84 40N A

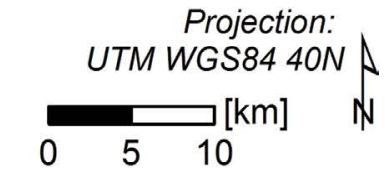

Figure 6: Landsat-8 (L-8) true color spectral reflectances, Sentinel-1 (S-1) SAR intensities and coherences for the period from June to October 2015 for test site B. 

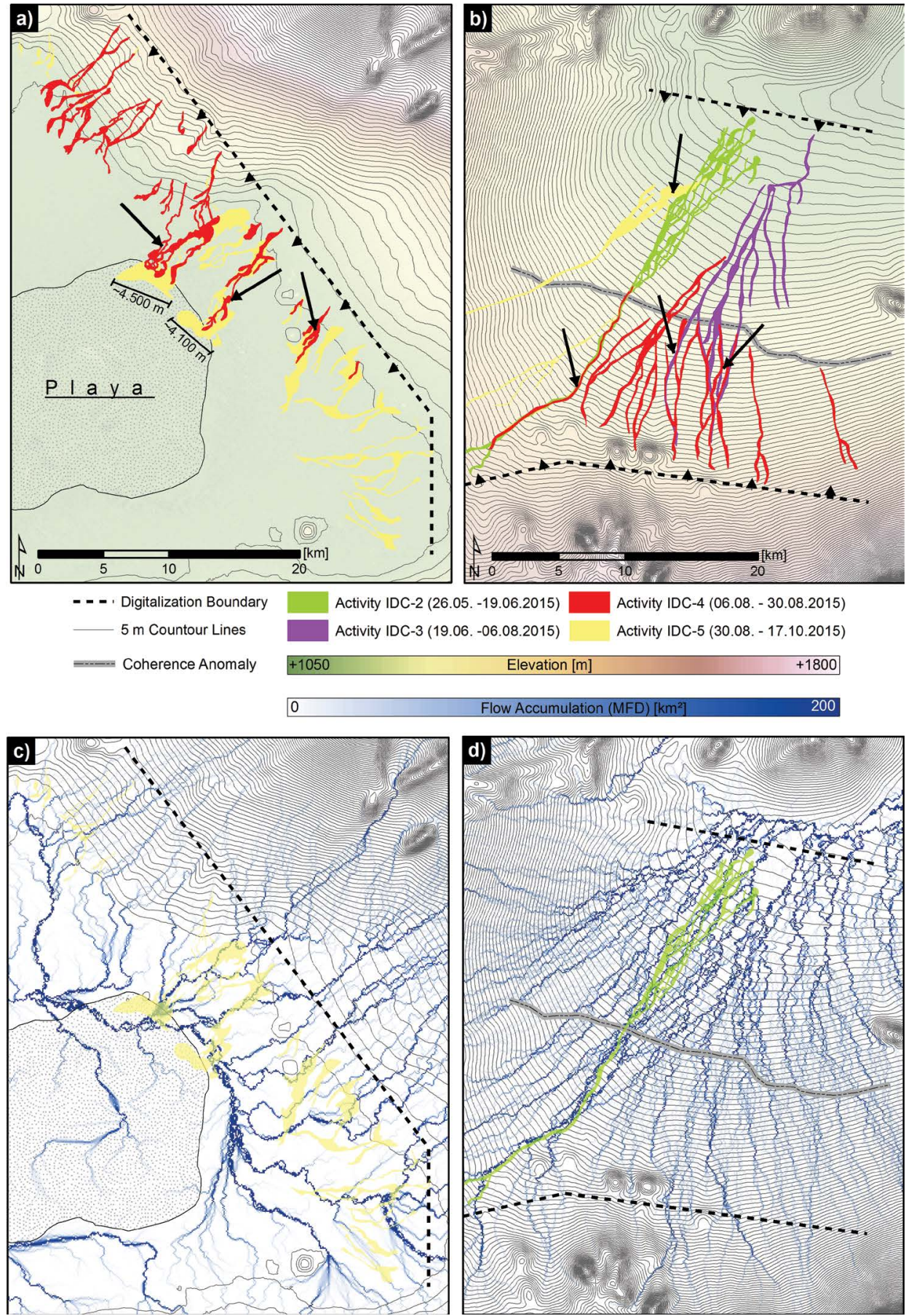

Figure 7: Detected changes and channel activity between May and October 2015 based on the analysis of InSAR coherences for a) test site $A ; b)$ test site B and flow accumulation derived from the SRTM digital elevation model via hydrographic modelling; c) test site A; d) test site B.

channels detected in IDC-2 and IDC-4 was partially congruent with the modelled channels, especially for the upslope locations that were characterized by steep terrain.

\section{Backscatter and spectral characteristics}

Three locations that showed high congruence between the recognized surface changes and the hydrographic model outputs were investigated in the following. Figure $8 \mathrm{a}$ shows the IDC-2 coherence and the channel that was digitized as active in IDC-2 (26.05 - 19.06.2015). A descriptive statistical assessment was carried out for the upper (Segment A) and lower (Segment B) part of the channel for the time series data of L-8, S-1 intensities and the S-1 coherences. Further, three cross sections were drawn for the upper (Cross Section 1), middle (Cross Section 2) and lower (Cross Section 3) course of the channel. The (Figure 8b, Figure 8c and Figure 8d) shows the IDC2 coherence values alone the cross sections from west to east. The activity of the channel caused a drop of the coherence in to order of -0.7 to -0.4 compared to the surroundings that showed high coherence values of about 0.6 to 0.9 . The variation of the coherence values was higher for the downstream location (Cross Section 3). The run off was concentrated as single channel till it reached the location close to the coherence anomaly. The runoff then diverged and widened and several separated small flows were visible in IDC-2.

It was examined how this activity could be delineated over time in the S- 1 and the L- 8 data and the statistics of the two segments were investigated for the time series data. The analyses of the S-1 intensities showed no noticeable variations of the SAR signals, neither for Segment A, nor for Segment B (Figure 8e and Figure 8f). The mean 


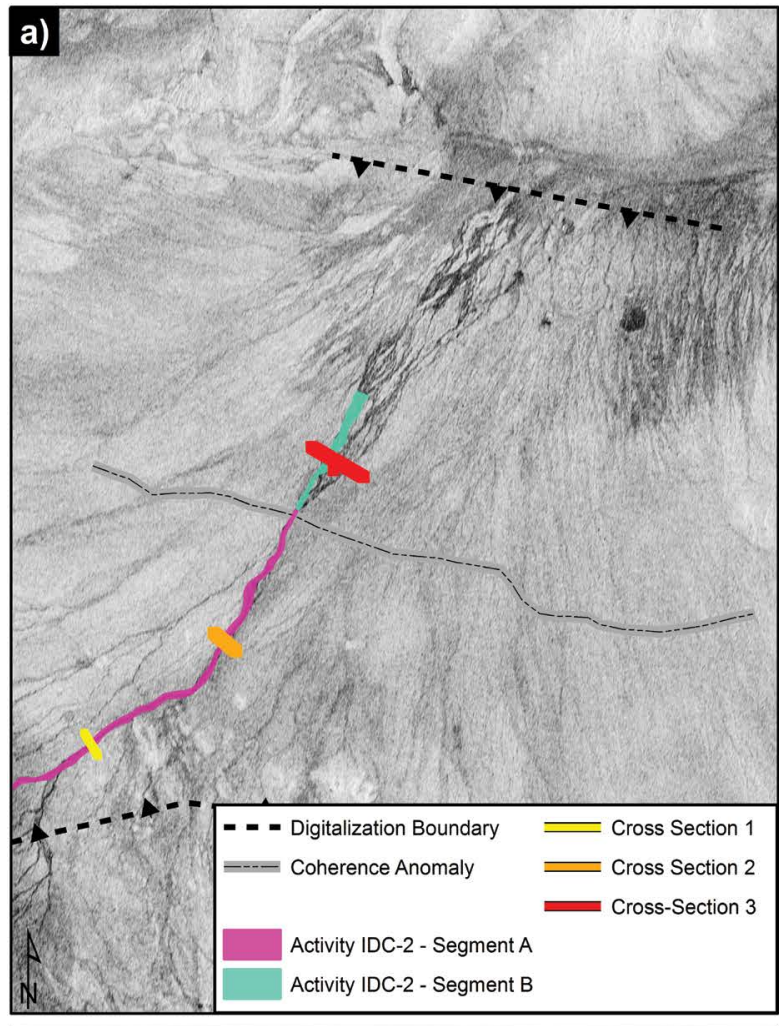

0.0

Coherence

1.0

e) Segment A - S-1 Intensities
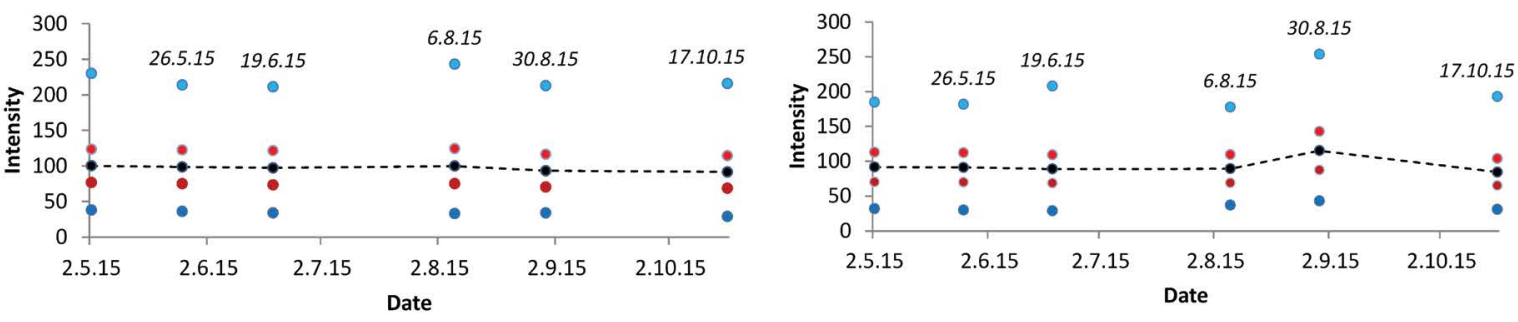

g) Segment A - S-1 Coherences

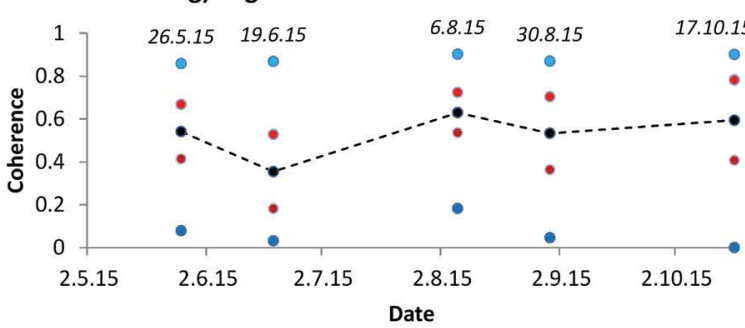

i) Segment A - L-8 Ratio (Red/Blue)
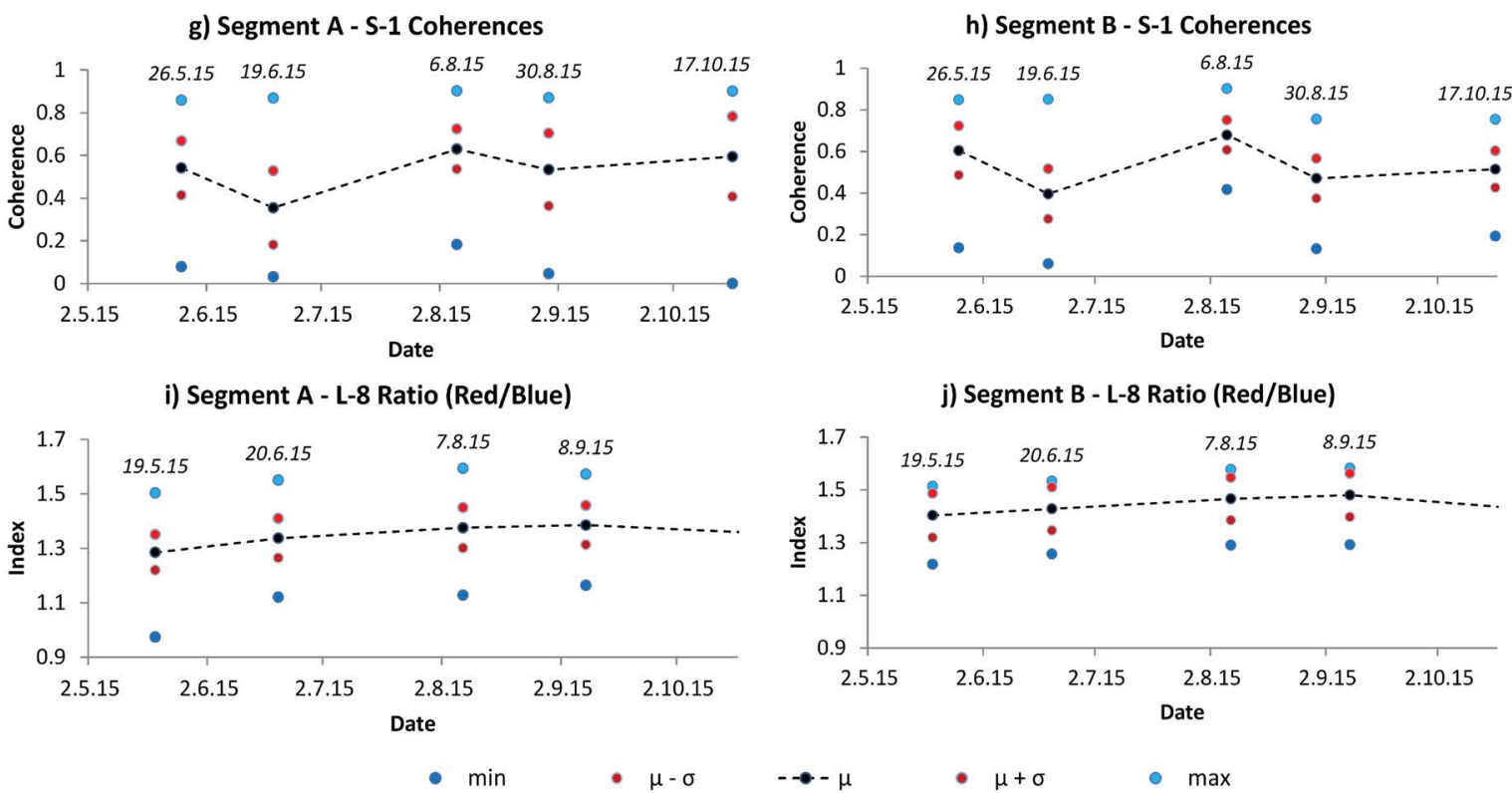

j) Segment B - L-8 Ratio (Red/Blue)
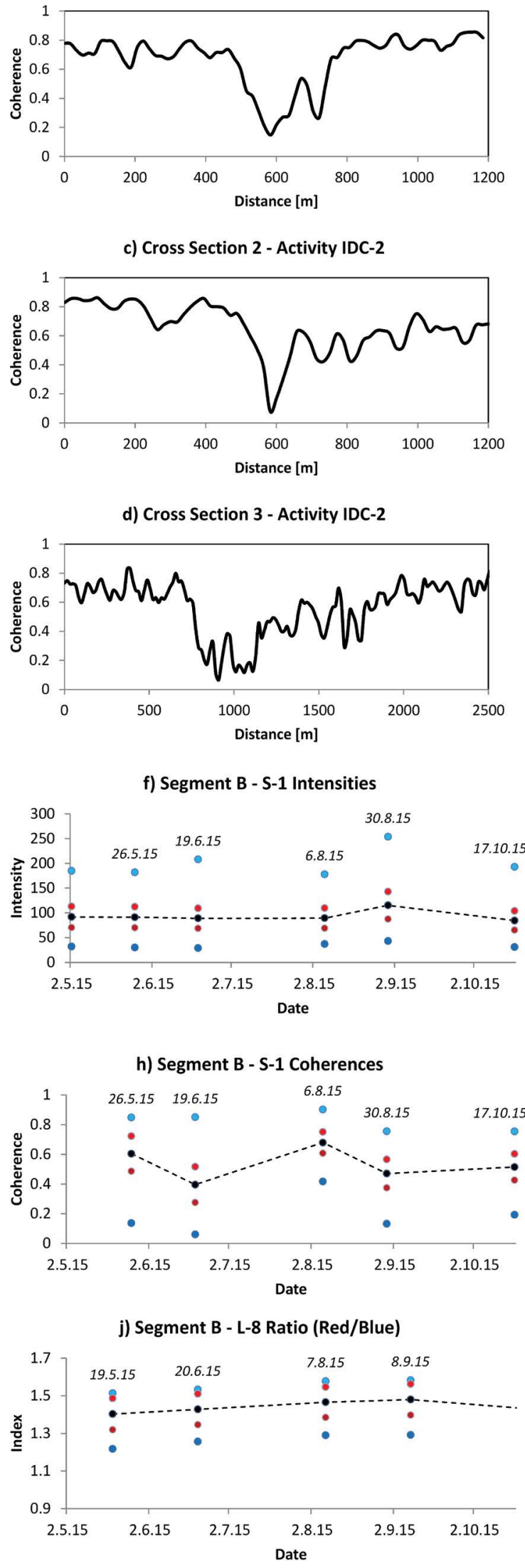

c) Cross Section 2 - Activity IDC-2

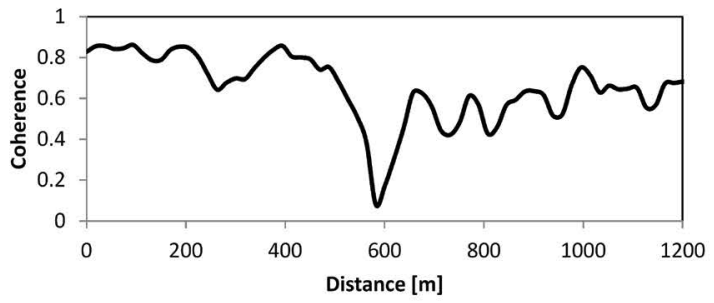

d) Cross Section 3 - Activity IDC-2

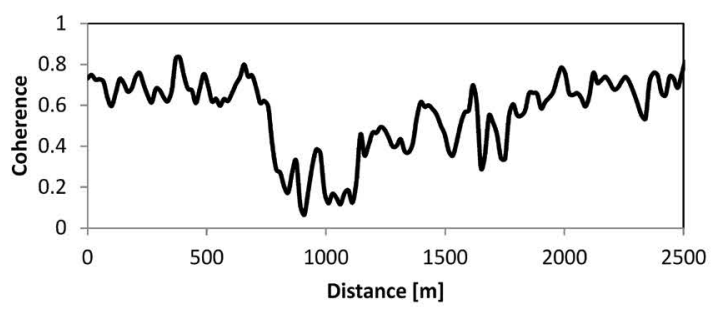

f) Segment B - S-1 Intensities along the cross sections 1,2 and 3 . Time series analyses of the segments A and B; e,f) Sentinel-1 (S-1) intensities; g,h) S-1 coherences; i,j) ratio between red and blue surface reflectance of Landsat-8 (L-8) data. 


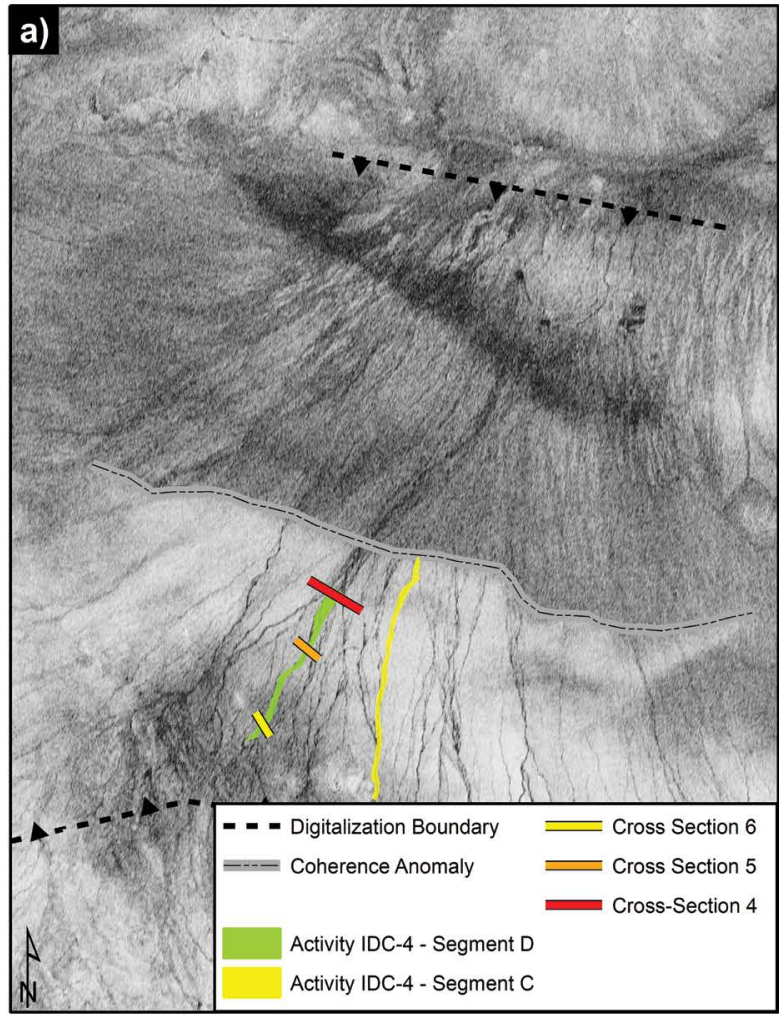

0.0

Coherence b) Cross Section 4 - Activity IDC-4

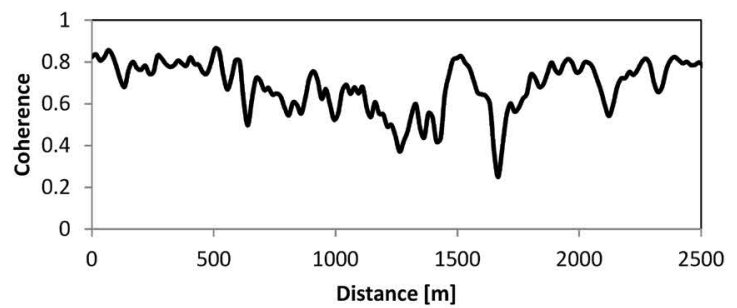

c) Cross Section 5 - Activity IDC-4

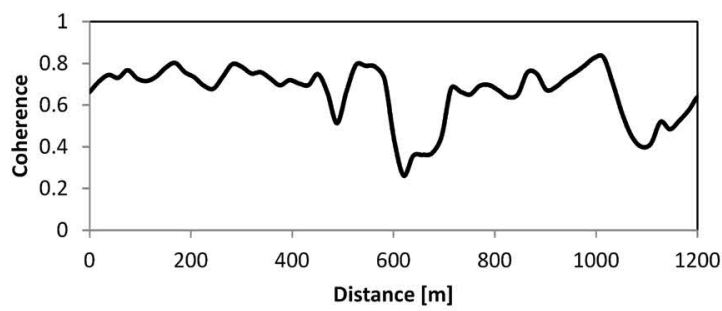

d) Cross Section 6 - Activity IDC-4
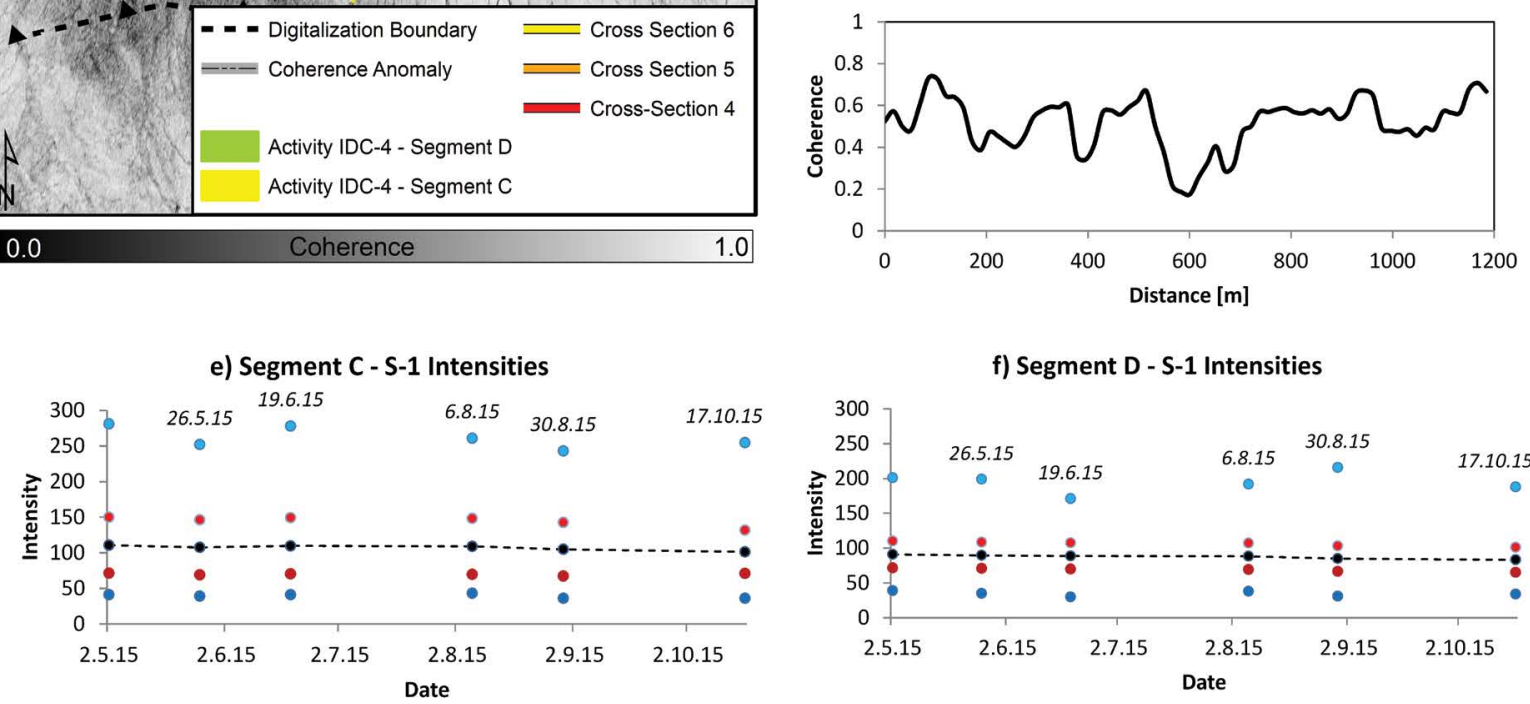

g) Segment C - S-1 Coherences

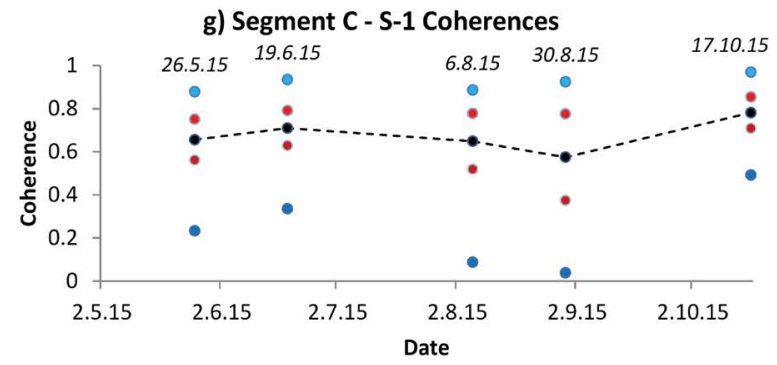

i) Segment C - L-8 Ratio (Red/Blue)

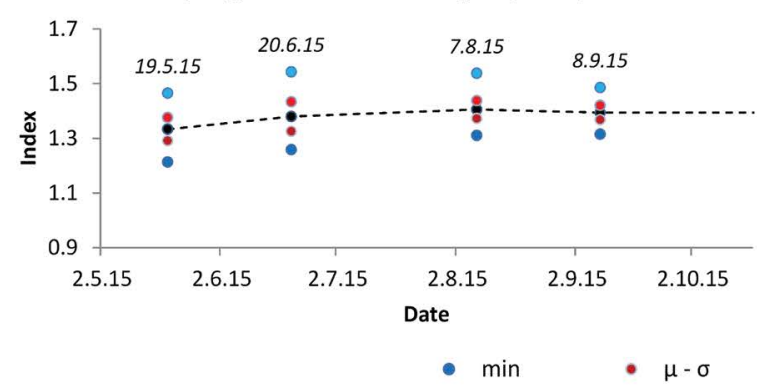

f) Segment D - S-1 Intensities

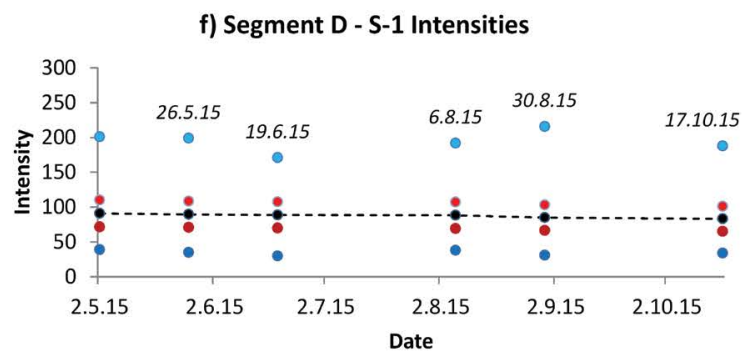

h) Segment D - S-1 Coherences

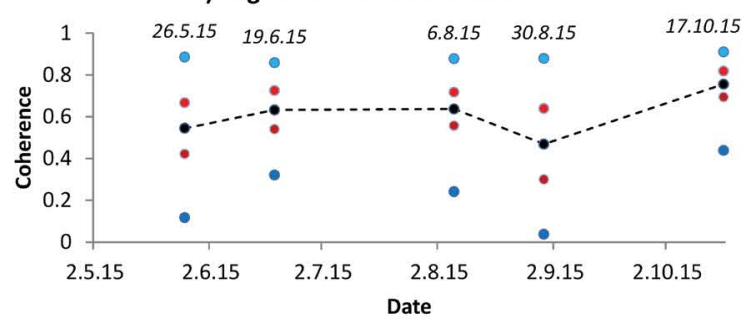

j) Segment D - L-8 Ratio (Red/Blue)

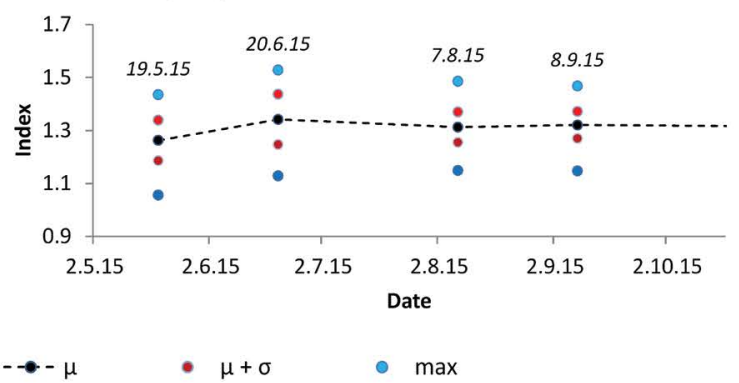

Figure 9: Assessment on the channel activity. a) IDC-4 coherence and location of the investigated segments and cross sections; b,c,d) IDC-4 coherence values along the cross sections 1, 2 and 3 . Time series analyses of the segments A and B; e,f) Sentinel-1 (S-1) intensities; g,h) S-1 coherences; i,j) ratio between red and blue surface reflectance of Landsat-8 (L-8) data. 
values were nearly constant over time and around 100 (DN), with standard deviations of about $25(\mathrm{DN})$. Contrary, the coherences showed reduced values for IDC-2 (19.06) and for both segments (Figure 8g and Figure 8h). The lowering of the coherence was in the order of -0.2 , respectively one standard deviation compared to IDC1 (26.05) and IDC-3 (06.08). Figure $8 \mathrm{i}$ and figure $8 \mathrm{j}$ show the ratio between the red and the blue channel of L-8 for the corresponding acquisition dates. The index values were nearly constant over time and were around 1.3 with standard deviations of about 0.15 . No variation was observable. The other bands of L-8 were analyzed in addition, but none of them showed a noticeable variation of the reflectance over time. Thus, the activity of the channel was neither noticeable in the S-1 intensities (ACD), nor in the L- 8 data, but only in the $S-1$ coherences $(C C D)$.

The same analyses were conducted for two channels that were digitized as active in IDC-4 (06.08 - 30.08.2015) in test site B. Figure 9a shows the S-1 coherence IDC-4 and the two channels marked as Segment $\mathrm{C}$ and Segment D. The figure further shows three cross sections that were drawn for the lower (Cross Section 4), middle (Cross Section 5) and upper (Cross Section 6) course of Segment D. Again the active runoff of the channel caused a decorrelation of SAR signals and the IDC- 4 coherence dropped in the order of -0.4 compared to the surroundings of the channel that showed coherence values of about 0.6 to 0.8 (Figure 9b, Figure 9c and Figure 9d) However, the variance was higher along the profiles and the location of the main channel was less clear compared to the situation in figure 8.

The analysis of the time series data showed that the S-1 intensities were not sensitive to the activity of the channel. The mean values were constant over time for both segments and were around 100-110 (DN). The standard deviations were in the order of 20-40 (DN) and no changes were noted for the dates that correspond to the IDC-4 coherences (Figure 9e and Figure 9f). The coherences showed lower values for IDC-4 (30.08.) and for both segments (Figure 9g and Figure $9 \mathrm{~h}$ ). The drop of the mean values was in the order of 0.2 to 0.3 . This lowering of the coherence corresponded to a difference of about one standard deviation compared to IDC-5 (26.05). Figure 9i and figure 9j show the ratio between the red and the blue channel of L-8 for the corresponding acquisition dates and both segments. The index values were nearly constant over time and were around 1.3 to 1.4 with standard deviations of about 0.15 . No variation was observable for the dates that correspond to the IDC- 4 coherence. The presence of runoff was only recognizable in the S-1 coherences (CCD), similar to the observations that were made for the channel segments of IDC-2 (Figure 8).

\section{Discussion}

The visual analysis of the time series data showed the general applicability of the ACD and CCD to indicate surface changes. Large scale events, such as the two flows in test site A, caused a noticeable alteration of the SAR intensities and coherences. Further, small scale channel activity was visual in the coherences, but not in the SAR intensities or the multispectral reflectances. Concerning the fluvial activity in test site B it is noticeable that different spatial patterns of channel activity were observed over time. The drainage and channel systems in test site B lied close together and in a distance of approximately $35 \mathrm{~km}$. Thus, one would intuitively expect a similar behavior and a spatial correlation. The observed differences may be a result of more distant upslope rainfall events that were locally restricted and not part of the study area and thus were not imaged by the sensor. It will be necessary to delineate the catchment areas to encounter this issue, e.g., via large scale modelling of hydrographic properties using DEM data, and to enlarge the test site. Such an assessment will further support the interpretation and help to find the forces that drove different spatial patterns. Along with this it will be necessary to compare the signal to higher quality reference data, e.g., data on the spatial-temporal distribution of the rainfall events, or in situ data on the activity/inactivity of the channels.

It is further of importance to notice the coherence anomaly that was observed between the southern and the northern part in IDC-4 and IDC-5. The distinct shape makes atmospheric effects unlikely, as well as locally restricted rainfall events. Possible explanations for this anomaly are differences of the grain sizes, or salinity. Both factors influence the development of soil moisture over time and therefore alter the SAR signal. This consideration is supported by the changing slope values: the terrain north of the line is steeper than the terrain south of the line. This topographic difference may indicate a different geological or sedimentary units and this possibility will be investigated in upcoming research via the comparison of the SAR data to in situ mapped geomorphology and sedimentology.

The activity of the channels over time was mapped via the SAR coherences and compared to the hydrographic model derived from digital elevation model data. The comparison of the model to the digitized surface changes showed that the detected runoffs were partially congruent with the modelled flow lines. This was especially true for upstream locations with rough topographic setting and for locations in sloped terrain. Contrary, major differences between the modeled and observed flow lines were found for low lying and flat terrain. Most likely this was caused by the preprocessing of the DEM and by the flow accumulation algorithms, which are known to be week in flat terrain. Further, the used elevation model had a spatial resolution of $90 \mathrm{~m}$ and thus was much coarser than the S-1 data. A better and more reliable hydrographic modelling will be possible with higher quality and resolution elevation data, e.g., in future with the TanDEM-X elevation model [28].

The statistical assessment was conducted for locations that showed high congruence between the recognized surface changes and the hydrographic model outputs. The first outcomes proved the applicability of the CCD. For all investigated channels it was found that neither the SAR intensities, nor the L-8 reflectances were sensitive to the runoff events. The intensities and surface reflectances were constant over time and no variations were found. Contrary, the statistics on the coherences indicated variations over time and these were associated with rainfall and runoff events - as far as we can judge from the reference data. The drop of the coherence after channel activity was distinct and in the order of -0.7 to -0.4 . Thus the CCD approach clearly indicated surface changes that were not detectable with ACD or multispectral imaging.

Nevertheless, it has to be pointed out here that the applicability of the CCD for mapping morphodynamic events is limited. First, it is unclear which coherence value should be used as threshold to indicate the activity of a channel and active runoff. To encounter this problem it will be necessary to collect independent (in situ) reference in order to train and adjust the change detector and to come to a quantitative assessment. Second, to detect channel activity via the coherence it is necessary that the surrounding uplands and the inactive parts of the drainage systems remain coherent. Extensive changes of the ground moisture regime will hinder the detection of activity via the CCD since moisture causes a decorrelation of SAR signals. Similar, the application of CCD is unsuited for vegetated areas and environments with dominant volume scattering. Therefore, the approach is restricted to arid and non-vegetated regions. Third, the influence of changing salinity and of chalk concentration on the coherence is unclear. As both have influence on the dielectric properties, changes in the concentration after rainfall events might alter the SAR signal, but the temporal dimensions and responses of these processes are unknown. Fourth, the temporal resolution of the data is crucial to indicate activity and changes. The longer the time lag between two acquisitions the more it is likely that the reference and test image will decorrelate. This means that a sufficient short time lag is needed to ensure a contrast between activity and inactivity. 
Additionally, the spatial resolution restricts the size of channels that can be detected with the shown approach. The S-1 IW data offer a spatial resolution in the order of 12-15 m, depending on the incidence angle and the orbit. Thus the size of the channels should be at least the size of one resolution cell, even though the CCD technique is sensitive to sub-pixel variations.

\section{Summary and Conclusion}

In this study Sentinel-1 (S-1) time series data were used for the detection of surface changes induced by fluvial activity in the arid region of Damghan Playa, Iran. Temporal corresponding Landsat- 8 (L-8) data were used to highlight differences between the spectral and the Synthetic Aperture Radar (SAR) data. The change detection techniques were based on the SAR imagery and two approaches were investigated: the Amplitude Change Detection (ACD) and the Coherence Change Detection (CCD). CCD is based on the analysis of the coherence that is calculated during the InSAR processing using the complex radar signals. CCD is known to be a sensitive feature to indicate surface disturbances and changes on sub-pixel level and has potential to indicate changes that are not noticeable in the spectral or SAR intensity data. Using this methods and data, the dynamics of the test site between the 02.05.2015 and the 17.10.2015 were investigated using six S- 1 and five L- 8 acquisitions. The weather data of a nearby climate station served as reference for rainfall and storm events that may have caused fluvial activity during the observation period. Further, a digital elevation model of the SRTM mission served as reference to indicate the topographic setting and the potential hydrographic situation.

The application of ACD and CCD was shown to be a promising way to detect the fluvial activity and short term morphodynamic events using SAR data. Major surface changes were revealed in the spectral, intensity (ACD) and coherence (CCD) data. Large scale changes were observed after strong rainfall and storm events in the east of Damghan Playa. The mapped changes indicated extensive material movement and accumulation- up to sizes of more than 4,000 $\mathrm{m}$ in width. Additionally, the CCD technique was capable to indicate small-scale channel activity of the drainage system. The fluvial activity between two observations caused a decorrelation of the SAR signals, due to phase changes. These alterations were most likely a result of material rearrangement and transportation in the bed of the channel.

An initial assessment of the time series data was carried out for two fluvial events that caused runoff and channel activity. A clear drop of the coherence in the order of $>-0.4$ was noticed, compared to the coherent surroundings and inactive parts. These inactive parts of the drainage systems and the uplands showed generally high coherence values $>0.7$ due to the arid nature of the site and the absence of vegetation. Further, the SAR signals of these regions were coherent over time; no matter if the repeat pass time lag was 24 or 48 days. The identified activities of the channels were neither recognizable in the spectral nor the SAR intensity data, but only in the coherence, as indicated by the descriptive statistics. The analysis for the entire time span highlighted the high dynamic and the complex tempo-spatial patterns of the fluvial activity of the test site. Consequently, the CCD is a promising technique to collect data on such complex activity. However, the shown approach misses so far a better quantification and independent reference data, e.g., information on true runoff events.

\section{Acknowledgments}

This publication was partially funded by the German Research Foundation (DFG) and the University of Wuerzburg in the funding program Open Access Publishing.
2. Alavipanahs K, Goossens R, Matinfar HR, Mohammadi H, Ghadiri M, et al. (2008) The Efficiency of Landsat TM And ETM+ Thermal Data for Extracting Soil Information in Arid Regions. J Agric Sci Technol 10: 439-460.

3. Zebker HA, Villasenor J (1992) Decorrelation in interferometric radar echoes. IEEE Transactions on Geoscience and Remote Sensing 30: 950-959.

4. Wright $P$, Macklin T, Willis $C$, Rye T (2005) Coherent Change Detection with SAR. $2^{\text {nd }}$ EMRS DTC Technical Conference, Edinburgh 17-20.

5. Preiss M, Stacy NJS (2006) Coherent Change Detection: Theoretical Description and Experimental Results. DSTO-TR-1851.

6. Scheuchl B, Ullmann T, Fifame K (2009) Change Detection using High Resolution TerraSAR-X Data - Preliminary Results. ISPRS Hannover Workshop, Germany.

7. Oyen AM, Koenders R, Aria SEH, Lindenbergh RC, Li J, et al. (2012) Application of synthetic aperture radar methods for morphological analysis of the Salar De Uyuni distal fluvial system. IEEE International Geoscience and Remote Sensing Symposium 3875-3878.

8. Bodart C, Ozer A (2007) The Use Of SAR Interferometric Coherence Images to Study Sandy Desertification in Southeast Niger: Preliminary Results. Proceedings of the Envisat Symposium, Montreux, Switzerland, 1-5.

9. Baade J, Schmullius C (2011) Monitoring Micro-Relief Morphodynamics Using TerraSAR-X Repeat-Pass Spotlight Images. 3th TerraSAR-X Science Team Meeting, DLR Oberpfaffenhofen 1-6.

10. Wegmuller U, Strozzi T, Farr T, Werner CL (2000) Arid land surface characterization with repeat-pass SAR interferometry. IEEE Transactions Geoscience and Remote Sensing 38: 776-781.

11. Liu JG, Black A, Lee H, Hanaizumi H, Moore J MCM (2001) Land surface change detection in a desert area in Algeria using multi-temporal ERS SAR coherence images. Int J Remote Sensing 22: 2463-2477.

12. Plank S (2014) Rapid Damage Assessment by Means of Multi-Temporal SAR - A Comprehensive Review and Outlook to Sentinel-1. Remote Sens 6: 4870-4906.

13. Balzter H, Cole B, Thiel C, Schmullius C (2015) Mapping CORINE Land Cover from Sentinel-1A SAR and SRTM Digital Elevation Model Data using Random Forests. Remote Sens 7: 14876-14898.

14. WDC (World Data Center for Meteorology) (2013) Climate Normals - Climate of the World.

15. Aghanabati A (2004) Major Sedimentary-Structural Units of Iran. 23 Geosciences conference, Geological Survey of Iran, Tehran.

16. USGS (2016a) Earth Explorer.

17. ESA (2016) Sentinels Scientific Data Hub.

18. ESA Sentinel Online (2016) Sentinel-1 Technical Guide.

19. Geudtner D, Torres R, Snoeij P, Davidson M, Rommen B (2014) Sentinel-1 System capabilities and applications. IEEE International Geoscience and Remote Sensing Symposium 1457-1460

20. USGS (2016b) Landsat 8 OLI (Operational Land Imager) and TIRS (Thermal Infrared Sensor).

21. EXELIS (2016) Atmospheric Correction - Dark Subtraction.

22. NOAA (2016) Aviation Weather Center.

23. Richards JA (2009) Remote sensing with imaging radar. Springer, Signals and Communication Technology 1-361.

24. Abubakr AAA-S, Biswajeet $P$, Sinan JH, Neda M (2013) Revisiting Methods and Potentials of SAR Change Detection. Proceedings of the World Congress on Engineering III: 2231-2237.

25. Touzi R, Lopez A, Bruniquel J, Vachon PW (1999) Coherence Estimation for SAR Imagery. IEEE Transactions on Geoscience and Remote Sensing 37: $135-149$

26. Wang L, Liu $H$ (2006) An efficient method for identifying and filling surface depressions in digital elevation models for hydrologic analysis and modeling International Journal of Geographical Information Science 20: 193-213.

27. Gruber S, Peckham S (2009) Land-Surface Parameters and Objects in Hydrology. In: Hengl T, Reuter H, Geomorphometry- Concepts, software, applications. Developments in soil science 1-772.

28. Krieger G, Moreira A, Fiedler H, Hajnsek I, Werner M, et al. (2007) TanDEM-X: a satellite formation for high resolution SAR interferometry. IEEE Transactions on Geoscience and Remote Sensing 45: 3317-3341.

\section{References}

1. Millington AC, Drake NA, Townshend JRG, Quarmby NA, Settle JJ, et al (1989) Monitoring salt playa dynamics using Thematic Mapper data. IEEE Transactions on Geoscience and Remote Sensing 27: 754-761. 